\title{
Tools and techniques for modelling production systems
}

\author{
C. R. McLean \\ Manufacturing Systems Engineering \\ National Institute of Standards and Technology \\ Gaithersburg, MD 20899 USA \\ (301)975-3511,FAX(301)257-9549, charles.mclean@nist.gov
}

\begin{abstract}
Industry needs software tool kit environments that integrate functions performed by engineers that are responsible for developing production systems. These tool kits would help engineers be more productive in the process of conceiving, designing, modelling, evaluating, and planning the implementation of manufacturing systems. Although individual tools are available to perform some of these functions, integrated tool kits are not. The lack of common information models and interface standards inhibits the development of interoperable software tools. The Production System Engineering (PSE) project at NIST is working with U.S. industry, academia, and a number of international collaborators to address this problem. This project is a part of the larger Systems Integration for Manufacturing Applications (SIMA) Program underway at NIST. The project team, working with the Black and Decker Corporation, selected a mitre saw as a test case for production system engineering. The mitre saw assembly line has been used to
\end{abstract}


identify functional and data requirements for integrating engineering tools. This paper will present our progress in developing modelling techniques, software tools, and tool kit interfaces for production systems engineering.

\section{Keywords}

Manufacturing, production systems engineering, software tool kits, integration interfaces

\section{TECHNICAL OBJECTIVES}

Production systems engineering (PSE) is a project area within the Systems Integration for Manufacturing Applications (SIMA) Program at NIST, see Barkmeyer (1995). The PSE goal is to develop solutions for integrating engineering software applications that are used to design, plan, and implement production systems for discrete parts manufacturing. The initial focus for the project is the engineering of small assembly lines for electromechanical product production. Objectives of the project include:

- Develop architectures, information models, database structures, interfaces, and techniques for integrating production system engineering tools, e.g., process specification, plant layout, and manufacturing simulation systems,

- Develop new database structures and exchange file formats, or, as appropriate, identify suitable existing formats for maintaining and sharing production system engineering data,

- Integrate commercial software applications into prototype tool kit environments using those database and exchange files to evaluate architectures and interface solutions,

- Test and validate interfaces in the prototype integrated system using real world data,

- Recommend potential standards based upon project results, i.e., information models for the relevant data, database structures for common databases, functionality of software tools, interfaces between tools and other system elements.

The benefits realised from the ability to implement integrated tool kits for production system engineering will be applicable to a broad sector of manufacturing industry. Architectures and interface solutions will help industry to rapidly integrate commercial off-the-shelf software applications. Tool kit technology will help engineers become more productive and reduce the cycle required to develop new production systems. Integrated tool kits will also help engineers identify design errors, make better decisions and more quickly evaluate the effects of those decisions.

By improving process specification and simulation capabilities, a much greater percentage of products will be produced correctly the first time. Better utilisation 
of production resources may be expected. The best resources for the job will be selected more often and less time will be consumed by non-productive work, e.g., producing scrap and performing rework. Furthermore, the overall time to perform the engineering function will also be reduced if fewer changes to plans and programs are required once a product goes into production. These improvements will result in fewer scrapped parts and less re-work. The integration of software packages and common databases will ensure that less time is wasted re-entering the same data into multiple engineering tools.

This paper presents an overview of the production system engineering activity model and the interfaces required to integrate a PSE tool kit. For more information on this topic, see McLean (1997).

\section{PRODUCTION SYSTEM ENGINEERING ACTIVITY MODEL}

Production systems that may eventually be engineered using tool kit environments include transfer lines, group technology cells, automated or manually-operated workstations, customised multi-purpose equipment, and entire plants. The initial focus and test case selected for this project is a small production line used to assemble power tools.

Examples of the production system engineering functions that have been addressed by the PSE Project include: 1) identification of product specifications and production system requirements, 2) modeling and specification of manufacturing processes, 3) plant layout and facilities planning, 4) simulation and analysis of system performance, 5) specification of interfaces and the integration of information systems, 6) task and work place design, and 7) management, scheduling and tracking of projects.

The interoperability of the commercial engineering tools that are available today is extremely limited. As such, users must re-enter data as they move back and forth between different software applications carrying out the engineering process. A long term objective of the project is to improve the productivity of users by specifying interfaces that enable the creation of an integrated environment where changes to data and decisions automatically percolate (flow seamlessly) through the various software applications. The primary mechanism that is currently being used to integrate software applications is file exchange, see ISO 10303-21 (1996). Information models for data to be exchanged are being developed using the EXPRESS modelling language, see ISO 10303-11 (1994).

A process model has been developed to identify some of the functions involved in production system engineering and the data required to integrated engineering software applications. The process model defines the functions that tools must perform in order to engineer a production system. The model also defines inputs, outputs, controls, and mechanisms for carrying out the functions. The process model is a key reference for defining the data flows and interfaces. A larger 
reference model has been developed for the entire SIMA Program, see Barkmeyer (1996).

The process model for production system engineering has been developed using Integrated Definition Method (IDEF0) modelling techniques, see MetaSoftware (1994). The model defines the tool kit functions and data inputs/outputs for each function. The top level of the model identifies the production system engineering function, its inputs, and its outputs. The first level of the model decomposes the engineering process into five major functions or activities listed below:

- Define the production system-engineering problem,

- Specify production processes required to produce the product,

- Design the production system,

- Model the system using simulation and evaluate its performance under expected operating conditions.

- Prepare project plans and budgets.

Lower levels of the model further decompose these activities, see McLean (1995). Detailed information models are under development to specify each data input and output identified in the process model. The information models are being used to implement shared-databases, exchange files, messages, and program calls for passing information between the commercial software tools.

\section{PSE TOOL KIT INTERFACES}

An initial set of interface specifications has been identified that would enable the integration of tools performing functions contained within the PSE process model. Required specifications are being developed either as PSE project efforts, as parts of other SIMA projects, or as ongoing international standards activities. The SIMA-based interfaces are being developed according to the Initial Manufacturing Exchange Specification (IMES) methodology, see Kemmerer (1997). The interface specifications that are required for an PSE initial tool kit include:

- Production system requirements.

- Product design.

- Assembly process specification.

- Plant layout.

- Production resource models.

- Production system evaluation.

- Engineering management data.

Each of these interfaces is briefly described below. 


\section{Production system requirements}

This specification will describe a model for the information that is required to fully describe a production system engineering problem. It will define the constraints that characterize the production system that is to be designed. A standard model for production system requirements data will ultimately enable application developers to access common or shared databases that describe the intended system. This data includes: product identification data and key product attributes, production system and engineering project type, manufacturing constraints and issues, critical milestone dates and schedules, expected or estimated costs, and identification of manufacturing data for related products. The PSE Project is developing this specification.

\section{Product design}

These specifications are used to describe the product to be manufactured, components, production resources, and other physical objects within the production system itself. Product designs contain various types of data including: product structure or bill of materials, geometry, topology, tolerances, and configuration management data. The specifications must provide neutral, common structures and information models for describing and accessing product data, e.g., ISO 10303 Standard for the Exchange of Product Model Data, commonly referred to as STEP. In developing solutions to integrate production system engineering applications, STEP is the preferred specification for representing product data. Initial Graphics Exchange Specification (IGES) and the file formats associated with specific $\mathrm{CAD}$ applications may also be used.

\section{Assembly process specification}

This specification will provide an interface for defining the product assembly process. Information contained within the process specification includes: process identification, process resources, process time and cost, and process relationships. Other process data that may be part of this specification includes:

- Activity relationship matrices are defined that describe how different processes relate to each other, e.g., required proximity or location

- Specification of requirements for processes, tooling, job skills, timing and line balancing, quality control, process audits, production yield,

- Development of process and inspection plans, process description sheets,

- Development of time standards for operations,

- Ergonomic analyses of manual tasks,

- Value engineering analysis (i.e., determination of job activities or steps that can be eliminated).

The PSE Project is developing this specification. 


\section{Plant layout}

This specification will define an interface for conveying manufacturing layout information between design and simulation software applications. Examples of information contained within layouts includes: site or facility identification data, drawing annotations, dimensions and tolerances, reference points, location and orientation of process machinery (both two dimensional footprints and solid models), material handling systems, tooling, fixture devices, safety systems, human ergonomic models, work areas, storage areas, aisles, building structural elements, utilities and services, and layer naming conventions within drawings and models. The work done under ISO 10303 STEP Application Protocol 227 Process Plant Spatial Configuration has provided a basis for developing an application protocol specification for manufacturing plant layout, see ISO 10303-227 (1995). The PSE Project is developing a specification for manufacturing plant layout.

\section{Production resource models}

This specification will define information models for representing production resources. It will identify the types of production resources and attributes required to support assembly. Production resources nominally include machinery, test systems, material handling devices and carriers, tooling, work holding devices, materials, and staff. These models must not only characterize the attributes of production resources, but also enable encapsulation of their geometry and behaviors to support simulation. Specifications initiated under the NIST Advanced Technology Program Rapid Response Manufacturing (RRM) Project in the area of manufacturing resource models will provide a foundation for the specifications needed in this area, see Jurrens (1995). The specifications developed under RRM will need to be extended to support additional data required to support assembly operations and construct manufacturing simulations.

\section{Production system evaluation}

This specification will define information models for representing the data applicable to the evaluation of the design of a production system. Examples of evaluation data include cost, performance measures, system reliability, safety, ergonomics, and timing data that is either associated with a particular production system or industry benchmark data used for reference and comparison purposes. Cost data may include: system design, engineering and construction costs, training costs, material costs, consumable costs, utility costs, unit production cost, touch labor cost per unit, estimated system maintenance costs. Performance measures include process yield, throughput, machine utilization, cycle time, work-inprocess, and inventory turnover rates. Reliability data include machine downtime, mean-time-between-failures, and mean-time-to-repair systems. Safety data may include identification of processes and chemicals that are hazardous to employees, systems, or products. Ergonomic data identifies the level of physical effort and/or 
special physical abilities are needed to perform the production tasks. The PSE Project is developing this specification.

\section{Engineering management data}

This specification will define the project management and cost data required to support the engineering of production systems. Project planning data includes: project phases, tasks, resources, and timing data (early/late start and end dates, estimated task durations, slack and float, and lead times.) Budget and cost data may include: project phase, planning, labor, tooling, capital equipment, projected maintenance, information and control system, operational, training, licensing and inspection, construction, installation, material (components, consumables), overhead (utilities, labor multipliers, area usage), and rental costs. Engineering management data will also reference and/or be associated with production system engineering data outlined in the models and interfaces that were previously described. The PSE Project is developing this specification.

\section{CONCLUSION}

If manufacturers are to remain competitive, they must not only continue to improve existing products, butoffer new ones. They must also strive to continuously improve its engineering processes and production systems. There are major opportunities to better utilize information technology in the design and implementation of manufacturing systems.

The PSE Project is working on the development of interface specifications and standards enabling the integration of production system engineering applications. These specifications could have a major impact on reducing the time and costs involved in bringing new products to market. Standard interfaces would help to formalize the production system engineering process and turn what is now more often an art, into a science. Seamless interoperation of plug-compatible software applications developed by different vendors could become a reality, thereby reducing software integration costs. Reduced integration costs would open the market for these products to a greater number of small and medium-sized manufacturers.

Standardization of several major types of information will be necessary to achieve this goal. These standards can only be achieved through the cooperation of software vendors, industrial users, researchers, and standards organizations. The Initial Manufacturing Exchange Specifications (IMES) documents developed by the SIMA Program at NIST will establish baseline interfaces and help fast track the development of these standards.

Work described in this paper was sponsored by the NIST Systems Integration for Manufacturing Applications (SIMA) and U.S. Navy Manufacturing Technology Program. No approval or endorsement of any commercial product by the National 
Institute of Standards and Technology is intended or implied. The work described was funded by the United States Government and is not subject to copyright.

\section{REFERENCES}

Barkmeyer, E.J.,[ed.] (1996),: "SIMA Reference Architecture, Part 1: Activity Models," NISTIR 5939, National Institute of Standards and Technology, Gaithersburg, MD, 1996.

Barkmeyer, E.J., Hopp, T.H., Pratt, M.J., Rinaudot, G.R., editors (1995) Background Study: Requisite Elements, Rationale, and Technology Overview for the Systems Integration for Manufacturing Applications Program, NISTIR 5662, National Institute of Standards and Technology, Gaithersburg, MD.

ISO 10303-11 (1994). Industrial automation systems and integration - Product data representation and exchange - Part 11: Descriptive methods: EXPRESS reference language manual, ISO, Geneva, Switzerland.

ISO 10303-21 (1996). Industrial automation systems and integration - Product data representation and exchange - Part 21: Implementation Methods: Clear text encoding of exchange structure, ISO, Geneva, Switzerland.

ISO/CD 10303-227 (1995). Industrial automation systems and integration Product data representation and exchange - Part 227: Application Protocol: Plant Spatial Configuration, ISO, Geneva, Switzerland.

Jurrens, K.K., Fowler, J.E., Algeo, M.E.A. (1995), "Modeling of Manufacturing Resource Information: Requirements Specification," NISTIR 5707, National Institute of Standards and Technology, Gaithersburg, MD.

Kemmerer, S. (1997), "Initial Manufacturing Exchange Specification (IMES): IMES Concept Document For Manufacturing Systems Integration," Draft NISTIR, National Institute of Standards and Technology, Gaithersburg, MD (in review).

McLean, C.R. and Leong, S. (1995): "A Process Model for Product System Engineering," Proceedings of the IFIP WG 5.7 on Production Management Systems, Seattle, WA.

McLean, C.R. and Leong, S. (1997): "Industrial Need: Production System Engineering Standards," NISTIR 6019, National Institute of Standards and Technology, Gaithersburg, MD.

Meta Software Corp. (1994), Design/IDEF User's Manual and Tutorial For Microsoft Windows, Meta Software Corp., Cambridge, MA. 